\title{
Decoding spike train ensembles using the cooperative interaction between task-dependent cortical neurons Seif Eldawlatly*1, Mehdi Aghagolzadeh ${ }^{1}$ and Karim Oweiss ${ }^{1,2}$
}

\author{
Address: ${ }^{1}$ Electrical and Computer Engineering Dept., Michigan State University, East Lansing, MI 48824, USA and ${ }^{2}$ Neuroscience Program, \\ Michigan State University, East Lansing, MI 48824, USA \\ Email: Seif Eldawlatly* - eldawlat@egr.msu.edu \\ * Corresponding author
}

from Eighteenth Annual Computational Neuroscience Meeting: CNS*2009

Berlin, Germany. 18-23 July 2009

Published: 13 July 2009

BMC Neuroscience 2009, I0(Suppl I):P96 doi:I0.I |86/I47|-2202-I0-SI-P96

This abstract is available from: http://www.biomedcentral.com/I47I-2202/I0/SI/P96

(c) 2009 Eldawlatly et al; licensee BioMed Central Ltd.

\section{Introduction}

A fundamental goal in systems neuroscience is to understand the mechanisms underlying the distributed nature of sensorimotor encoding by populations of neurons. In this work, we examine to what extent the existence of taskdependent functional connectivity between cortical neurons plays a role in encoding task information. To identify the functional connectivity, we used Dynamic Bayesian Networks (DBN) that fit a probabilistic model to spike train data. We demonstrate that the inferred functional connectivity is consistently similar across repeated trials of the same task while consistently different across different tasks.

\section{Methods}

A center-out reach task was designed to simulate premotor neural activity during arm movement planning. The arm moved from a central point to one of eight targets equally distributed around a circle. A 2-layer network with 166 neurons total was simulated, where the firing probability of each neuron was conditionally Poisson given the stimulus and the firing history of other neurons connected to it. In the input layer, 66 neurons were directly tuned to three movement parameters: direction, speed, and end-point, with overlapped tuning widths and no inter-neuronal connectivity. In the integration layer, 100 neurons were modeled with no specific tuning to the movement parameters. Each neuron in this layer received connections from a random input layer subset of neurons. Initially, neurons in this layer were synaptically coupled to each other with fixed weights. These synapses were dynamically adjusted following a Spike-Timing Dependent Plasticity (STDP) rule, where connections between task-dependent/independent neurons were potentiated/ depressed. After the network was trained, no more STDP was permissible and the obtained network topology was kept fixed. We then used 100 trajectories per target to generate the spike trains for the analysis. We randomly selected ten neurons from the integration layer and used DBN to infer the connectivity among them for each trajectory.

\section{Results}

For the same target, the inferred networks across multiple repeated trials showed high degrees of similarity quantified in terms of the appearance frequency of the connections ( $>85 \%$ of the trials) for a given target. In contrast, networks inferred for different targets were consistently different. This demonstrates that task-information was embedded in the connectivity between integration layer neurons and that a probabilistic model of cooperative interaction between a subset of these neurons captured task information such that decoding discrete targets as well as continuous arm trajectory was successful with over $90 \%$ accuracy. 


\section{Acknowledgements}

This work was supported by NIH grant \#NS054 I48-0IAI.

Publish with Bio Med Central and every scientist can read your work free of charge

"BioMed Central will be the most significant development for disseminating the results of biomedical research in our lifetime. " Sir Paul Nurse, Cancer Research UK

Your research papers will be:

- available free of charge to the entire biomedical community

- peer reviewed and published immediately upon acceptance

- cited in PubMed and archived on PubMed Central

- yours - you keep the copyright 